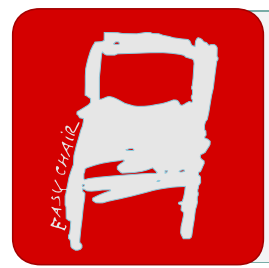

EPiC Series in Health Sciences

Volume 4, 2020, Pages 122-125

CAOS 2020. The 20th Annual Meeting of the International Society for Computer Assisted Orthopaedic Surgery

\title{
Automatic Markerless Tracking for Computer-Assisted Orthopaedic Surgery
}

\author{
Xue Hu, He Liu, and Ferdinando Rodriguez y Baena ${ }^{1}$ \\ Mechatronics in Medicine Laboratory, Imperial College, London, U.K. \\ xue.hu17@imperial.ac.uk, he.liu15@imperial.ac.uk, f.rodriguez@imperial.ac.uk
}

\begin{abstract}
In computer assisted orthopaedic surgery, it is important to find the correct spatial location of the target in a predefined world coordinate, so that the model can be transformed accordingly onto the surgical site for surgeons' reference. Current tracking systems mainly rely on the detection of optical markers inserted into the anatomy. The invasiveness of fixation pins increases operating time and bone complications. Automatic markerless tracking is therefore preferred in practice. In this paper, we integrate an automatic RGBD-image based segmentation neural network and a fast markerless registration algorithm to achieve the markerless tracking purpose. An experimental test with a metal leg was designed. By forcing the alignment of the measured hip joint centre, the overall tracking was shown to be sub-degree in terms of orientation accuracy, which is clinically acceptable.
\end{abstract}

\section{Introduction}

Considering cost, accuracy and mobility, optical tracking has become the main choice for many commercial navigation systems. Conventionally, as feature-based tracking is often noisy and computationally expensive, optically detectable markers (e.g., infrared-reflective spheres) are rigidly inserted into the target bone so that the target's location can be inferred from markers' movement. The marker preparation and insertion, however, can lead to human-induced errors [1], a longer workflow [2] and most importantly, a more evasive procedure that may cause infection, nerve injury, and bone fracture for patients [1].

Instead of performing a manual initial registration and updating the transformation based on marker tracking results, markerless tracking can be automatically achieved by a fast registration between a reference model and the real-time segmented target in world space. One of our previous researches has shown that a trained neural network can successfully segment the target femur from a RGBD video, with intersection over union of up to 0.87 [3]. The aim of this paper is to complete the markerless tracking process by applying an accurate and fast registration algorithm to these segmentation results. An experimental trial with a synthetic limb was designed to simulate a knee surgery procedure and to evaluate tracking performance. Because of the imperfect camera sampling, noise is inevitable on the distal femur, rendering the registration result, especially the rotational angle, inaccurate. For better accuracy, we adopted and compared two registration algorithms, a point-to-plane iterative closest point (ICP) registration [4] and a bounded ICP [5] method. 


\section{Materials and Methods}

Fig. 1 shows the system setup. An optical tracker (FusionTrack500, Atracsys LLC) was regarded as the world space. Before online tracking, the target femur was scanned with an HDI Compact 3D Scanner to obtain the reference model $F_{M}$. An array of optical markers $M_{1}$ was attached to a RGBD camera (RealSense D415, Intel Corp.). The coordinate transformation between $M_{1}$ and the camera was calibrated. For implementation of the bounded-ICP method, the hip centre in world space $H_{A}$ needs to be measured in advance. An optical marker was attached to the leg and the surgeon was asked to randomly rotate the limb. The movement of the marker was tracked to calculate the centre of rotation by means of the Pratt sphere fitting algorithm [6]. In practice the modelled hip centre $H_{M}$ can be directly obtained by pre-operative Computed Tomography (CT) imaging. In our experiment, the femur surface was instead manually scanned with an Atracsys probe, before it was registered against $F_{M}$. According to the resultant ${ }_{R}^{M T}$, the $H_{A}$ was transformed to $H_{M}$.

A $\mathrm{C}++$ registration programme ran in real-time on a computer with an IntelR@CoreTMi74790 processor and 16 gigabytes memory. No external graphic cards were used. The output of online segmentation is a point cloud where each point's intensity represents the possibility of that point belonging to the target. Points with intensity higher than a threshold (i.e., 0.8) were regarded as the measured femur points $F_{R}$. For the point-to-plane ICP method, intensities were also used as weights for the total registration error computed between $F_{M}$ and $F_{R}$ at every iteration. For the bounded ICP method, $H_{M}$ and $H_{R}$ were additionally required as an input. The registered femur pose in depth camera $F_{M}^{(D)}$ coordinates was then transformed to the global Atracsys frame by:

$$
F_{M}^{(A)}(t)={ }_{M_{1}}^{A} T(t) \times{ }_{D}^{M_{1}} T \times F_{M}^{(D)}(t)
$$

In order to establish the ground-truth for accuracy evaluation, a marker $M_{2}$ was inserted into the synthetic limb. The femur surface was manually probed. $F_{M}$ was registered accordingly, in order to obtain the initial femur pose. This pose was transformed back to local $M_{2}$ space. Based on the constant spatial relationship between $M_{2}$ and the target bone, the ground-truth pose in any time stamp can be obtained by:

$$
\begin{aligned}
F_{M}^{\left(M_{2}\right)}(t) & =F_{M}^{\left(M_{2}\right)}\left(t_{0}\right)={ }_{A}^{M_{2}} T\left(t_{0}\right) \times F_{M}^{(A)}\left(t_{0}\right) \\
F_{M}^{(A)}(t) & ={ }_{M_{2}}^{A} T(t) \times F_{M}^{\left(M_{2}\right)}(t)
\end{aligned}
$$

\section{Results}

The point-to-plane ICP and Bounded-ICP can run at a frame rate of 5-6 Hz and 3.5-5 Hz, respectively. The figure shows a comparison of the averaged tracking error between the two methods. It can be seen that they have an equivalent translational error of $6 \mathrm{~mm}$ in total. The rotational error, hoever, is effectively reduced by the bounded-ICP algorithm, to under 1 degree. The variation of the bounded-ICP method, however, is much larger. This is because the algorithm uses fewer points so that it is especially vulnerable to the false positives in the segmentation data. 


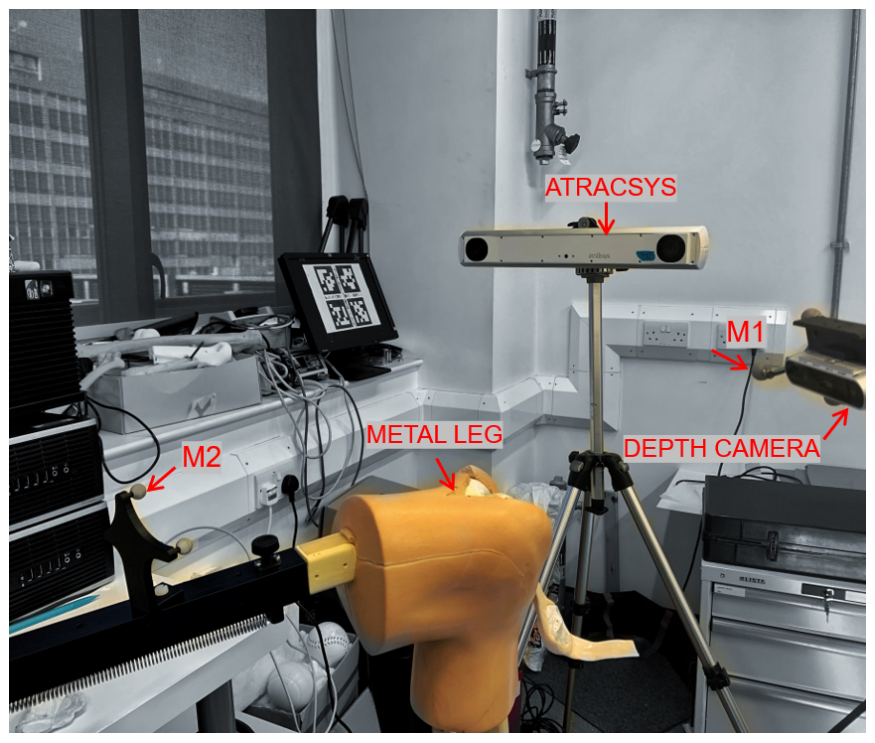

Figure 1: An overview of the system setup for automatic markerless tracking.
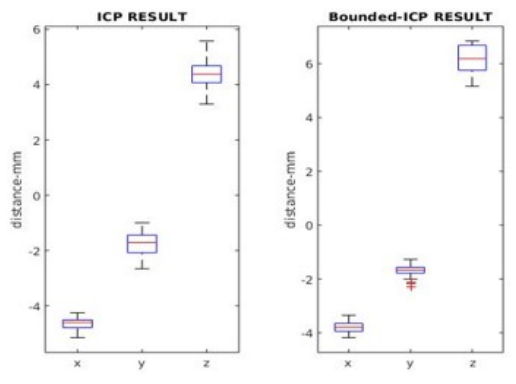

(a) 3D translational error
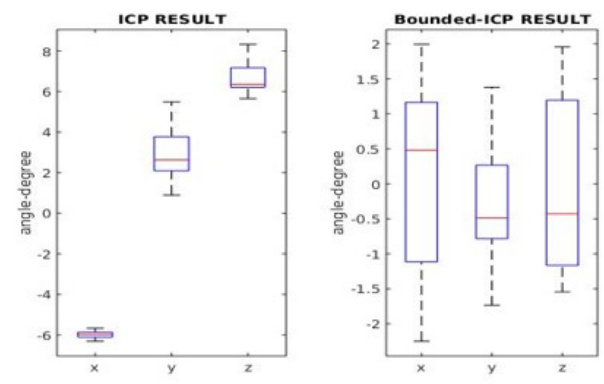

(b) 3D rotational error in coronal plane(x), sagittal plane $(y)$ and transverse plane $(z)$

Figure 2: Comparison of tracking errors between two registration methods.

\section{Discussion}

In this paper, we proposed an automatic markerless tracking method for computer assisted orthopaedic surgery. By integrating a pre-trained segmentation network and a novel registration technique, we show that the tracking can achieve a sub-degree rotational error, which is clinically acceptable [7]. For the practical usage, a 3D model can be obtained by the CT imaging and used for the pre-operative planning. Once captured by a commercial depth camera after the standard surgical exposure, the target femur can be recognised and registered with the model to guide the surgeon with useful information. The surgeon does not need to collect registration points manually or insert pins for tracking. Although the translational error is still large compared to the accuracy of $1 \mathrm{~mm}$ achieved by conventional registration [8], it is less important than orientation accuracy in tasks such as aligning a knee implant. Also, the inclusion of a GPU could drastically improve the speed of convergence in the future. The current tracking 
accuracy is mainly restricted by the quality of depth camera, segmentation network and offline calibration. Future research opportunities exist in optimising the neural network's structure and training to further improve accuracy. Another important issue to be addressed is the shape change of the tracked target caused by the operation. A registration method that can be adaptive with geometric changes is necessary for practical applications.

\section{References}

[1] Arndt P Schulz, Klaus Seide, Christian Queitsch, Andrea Von Haugwitz, Jan Meiners, Benjamin Kienast, Mohamad Tarabolsi, Michael Kammal, and Christian Jürgens. Results of total hip replacement using the robodoc surgical assistant system: clinical outcome and evaluation of complications for 97 procedures. The International Journal of Medical Robotics and Computer Assisted Surgery, 3(4):301-306, 2007.

[2] Don C Beringer, Jay J Patel, and Kevin J Bozic. An overview of economic issues in computerassisted total joint arthroplasty. Clinical Orthopaedics and Related Research@, 463:26-30, 2007.

[3] He Liu and Ferdinando Rodriguez Y Baena. Automatic markerless registration and tracking of the bone for computer-assisted orthopaedic surgery. IEEE Access, 8:42010-42020, 2020.

[4] Yang Chen and Gérard G Medioni. Object modeling by registration of multiple range images. Image Vision Comput., 10(3):145-155, 1992.

[5] Ferdinando Rodriguez y Baena, Trevor Hawke, and Matjaz Jakopec. A bounded iterative closest point method for minimally invasive registration of the femur. Proceedings of the Institution of Mechanical Engineers, Part H: Journal of Engineering in Medicine, 227(10):1135-1144, 2013.

[6] Vaughan Pratt. Direct least-squares fitting of algebraic surfaces. ACM SIGGRAPH computer graphics, 21(4):145-152, 1987.

[7] Matjaz Jakopec, F Rodriguez y Baena, Simon J Harris, Paula Gomes, Justin Cobb, and Brian L Davies. The hands-on orthopaedic robot" acrobot": Early clinical trials of total knee replacement surgery. IEEE Transactions on Robotics and Automation, 19(5):902-911, 2003.

[8] Shunsaku Nishihara, Nobuhiko Sugano, Miho Ikai, Toshihiko Sasama, Yuichi Tamura, Shinichi Tamura, Hideki Yoshikawa, and Takahiro Ochi. Accuracy evaluation of a shape-based registration method for a computer navigation system for total knee arthroplasty. The journal of knee surgery, 16(2):98-105, 2003. 